\title{
Usos y tendencias adictivas de una muestra de estudiantes universitarios españoles a la red social Tuenti: La actitud positiva hacia la presencia de la madre en la red como factor protector
}

\author{
Mercedes Durán* y José M. Guerra \\ Facultad de Psicología, Universidad de Sevilla
}

\begin{abstract}
Resumen: El objetivo de este trabajo fue estudiar, desde el paradigma de usos y gratificaciones, el uso que los estudiantes universitarios españoles ( $N$ $=221$ ) hacen de la red social Tuenti, y en qué medida estos usos se asocian con sus niveles de adicción a dicha red social. Además se analizó el papel que los progenitores tienen como facilitadores o inhibidores de dichas ten dencias adictivas y el impacto del género. Los resultados muestran cuatro categorías de usos de la red Tuenti: entretenimiento, comunicación a través del muro, comunicación a nivel privado e información social. Además, la actitud positiva hacia la presencia de la madre en la red Tuenti se muestra como un factor de protección de las tendencias adictivas a la red. Por último, se han encontrado diferencias estadísticamente significativas en función del género, tanto en los usos que realizan chicos y chicas, como en sus patrones de adicción a la red Tuenti, de tal manera que los chicos informan de niveles de adicción superiores a los informados por las chicas en el uso de esta red social.

Palabras clave: Tuenti; redes sociales; usos; adicción; factor protector.
\end{abstract}

\section{Introducción}

La expansión del uso de Internet ha supuesto toda una revolución social, en la medida que ha facilitado tanto el acceso a múltiples fuentes de información como al desarrollo de nuevos espacios de comunicación y convivencia. En estos nuevos lugares de interacción, los jóvenes han encontrado un espacio para su desarrollo social, emocional y cognitivo (Livingstone, 2008; Roberts, Foehr y Rideout, 2005), ocupando en algunos casos una parte importante de su tiempo de ocio (Kohut et al., 2011).

Una de las aplicaciones que mejor aceptación ha tenido, en este sentido, han sido las denominadas redes sociales o SNS (Social Networking Sites). Las redes sociales, tipo Facebook, MySpace, Bebo o Tuenti, permiten a sus usuarios desarrollar espacios compartidos, visibles para sus "amigos o contactos" en los que es posible crear perfiles públicos individuales, e interactuar tanto con amigos con los que se mantiene un contacto fuera de la red como con personas con las que no se mantiene una relación más allá de la propia red social (Kujath, 2011).

Las redes sociales, como han puesto de manifiesto diversos estudios, suponen para los jóvenes un escenario de oportunidades y riesgos (Livingstone, 2008). Así, el uso de redes sociales facilita el desarrollo de comportamientos positivos para la formación de la propia identidad (Bargh y McKenna, 2004), las conductas prosociales (Wright y Li, 2011), hábitos saludables (Lowe, Barnes, Teo y Sutherns, 2012) y el bienes-

* Dirección para correspondencia [Correspondence address]: Mercedes Durán Segura. Facultad de Psicología. Camilo José Cela s/n. 41018, Sevilla (España). E-mail: mduransegura@us.es
Title: Spanish university students' uses of the social network Tuenti and their addiction levels: The protective role of the positive attitude toward mothers' presence as contact.

Abstract: The present work aimed to study, from the uses and gratifications paradigm, the uses that Spanish university students $(N=221)$ do of the social networking site Tuenti, and the relationship between these uses and young's self-reported levels of addiction towards Tuenti. Also, the students' attitudes towards the presence of their parents as contacts in Tuenti, and the impact of gender were analyzed. The results showed four different categories of uses: entertainment, public communication, private communication and social information. Also, young's positive attitude toward the presence of the mother in Tuenti played a protective role in their addition levels towards this social networking site. Finally, statistically significant gender differences in the uses of Tuenti and also in their addiction levels toward Tuenti were found.

Key words: Tuenti; social networking sites; uses; addiction; protective fac-

tar de los propios implicados (Ellison, Steinfield y Lampe, 2007). A pesar de los numerosos beneficios asociados al uso de las redes sociales, es posible identificar también sucesos negativos asociados a su uso. Por ejemplo, celos y desconfianzas en relaciones de pareja (Muise, Christofides y Desmarais, 2009), violación de la privacidad (Tokunaga, 2011), bajo rendimiento académico (Kalpidou, Costin y Morris, 2011), ciberacoso (Lyndon, Bonds-Raacke y Alyssa, 2011), conflictos familiares (Gentzler, Oberhauser, Westerman y Nadorff, 2011) y cyberbullying (Kwan y Skoric, 2013; Ortega et al., 2012). Este lado negativo de las redes sociales, puede estar asociado al tipo de uso que los participantes desarrollan en la red social. Así, como apunta Becoña (2006), las redes sociales pueden "atrapar" a las personas, sobre todo adolescentes y jóvenes, ya que el mundo virtual contribuye a crear una falsa identidad y a distanciarlas o a distorsionar su mundo real, generando de esta manera un entorno propicio para el desarrollo de una conducta adictiva (Kuss y Griffiths, 2011).

Esta vinculación puede agravarse, aún más, cuando la persona cuenta con una red de contactos que propicie su aislamiento de entornos no digitales. En este sentido, diversos estudios han mostrado cómo algunas características de los "amigos" se relacionan con este patrón de aislamiento y adicción (Kalpidou et al., 2011). Autores como O’keeffe y Clarke-Pearson (2011) han alertado de la necesidad de que los padres tomen conciencia de la naturaleza, potencialmente peligrosa, de los medios digitales, y actúen para lograr un uso adecuado y saludable.

En esta línea, este estudio examina el uso que jóvenes españoles dan a la red social Tuenti, y en qué medida el tipo de uso se asocia a una tendencia adictiva a la misma. Además 
se analiza el papel que la figura materna puede jugar a la hora de reducir la dependencia frente a la red social. Por último, teniendo en cuenta los hallazgos de diferentes estudios que sugieren la existencia de diferencias de género en los usos de redes sociales (Chorng-Shyong, Shu-Che y Chih-Chien, 2011) incluimos la variable género con el objetivo de analizar la existencia de diferencias en función del género en los usos que los jóvenes dan a la red social Tuenti.

\section{Tipos de usos y gratificaciones en redes sociales}

El paradigma de usos y gratificaciones (Katz, 1959), proveniente de los estudios de mercado en radio y televisión, ha sido utilizado con éxito en el ámbito de las nuevas tecnologías (Cheung, Chiu y Lee, 2010; Raacke y Bonds-Raacke, 2008). Desde este paradigma se vincula el uso de determinados medios con la satisfacción de necesidades individuales, $y$ se asume que los usuarios dirigen sus conductas de manera consciente y teniendo presente cuáles son sus necesidades. Cada individuo selecciona los estímulos a los que quiere responder, atendiendo a causas como sus valores, intereses y funciones sociales. Por tanto, más que ser los medios los que dicen al usuario qué ver, son los usuarios de forma activa los que lo deciden, atendiendo a sus necesidades y la gratificación que les proporcionen. Si bien la investigación precedente apoya la suposición de que todo el consumo de los medios de comunicación requiere la participación activa de la audiencia (Ruggiero, 2000; Severin y Tankard, 1997), la propia naturaleza de los medios basados en Internet (los usuarios de forma activa deben hacer clic y escribir para poder acceder al contenido) hace que la conducta de estos usuarios sea más consciente y orientada hacia los objetivos de su consumo (LaRose y Eastin, 2004).

Algunos autores han comenzado, desde este paradigma, a analizar cuáles son las necesidades subyacentes al uso de las redes sociales, de manera individual (Cheung y Lee, 2009), grupal (Park, Kee y Valenzuela, 2009; Raacke et al., 2008), o como elemento de vinculación política (Ancu y Cozma, 2009), identificándose en dichos estudios que sería posible y útil diferenciar entre tipos de necesidades. Así por ejemplo, Cheung y cols. (2010), identificaron 5 categorías de necesidades para usuarios de la red social Facebook, pero solo 3 de ellas (mantenimiento de contacto interpersonal, mejora social y entretenimiento) se relacionan con la intención de participación conjunta.

A pesar de la extensión y uso de este paradigma, no existe hasta la fecha ningún estudio que haya analizado la red social Tuenti desde esta perspectiva, por lo que se desconoce cuáles son las necesidades que puede estar cubriendo la red social más utilizada entre los jóvenes españoles (SanchezFranco y Roldan, 2010), ni en qué medida alguna de esas necesidades se podría relacionar con conductas peligrosas como patrones adictivos en su uso. Como han indicado Kuss y Griffiths (2011) comprender las motivaciones que subyacen al uso de las redes sociales es esencial para comprender las tendencias adictivas en el uso de las redes sociales.

\section{Motivaciones y tendencias adictivas en el uso de re- des sociales}

La literatura precedente ha mostrado que las personas con signos de alta identidad social, telepresencia (sentirse presente en el entorno virtual) y altruismo tienden a utilizar en mayor grado las redes sociales porque las perciben como un recurso estimulante (Know y Wen, 2010). Este rasgo social ha mostrado ser incluso más explicativo que las motivaciones individuales de los usuarios (Kim, Kim y Nan, 2010). La utilización de las redes sociales como forma de estar en contacto con los amigos es una de las motivaciones principales de su uso (Subrahmanyam, Reich, Waechter y Espinoza, 2008). En este sentido, es el medio preferido por los estudiantes como forma de contacto -no presencial- e, incluso para algunos, es un medio más adecuado que los contactos presenciales o cara a cara (Kujath, 2011).

Como indican Kuss y Griffiths (2011), las redes sociales son utilizadas principalmente para el mantenimiento de las redes de contactos que los sujetos ya poseen previamente. La accesibilidad que conlleva el contacto mediante la red social, al permitir una comunicación tanto sincrónica -mediante chats integrados- como asincrónica -mensajería directa similar a los sms o distribuida mediante muros-, puede hacer que los usuarios se sientan obligados a mantenerse en contacto con sus redes a través de una red social, lo que como apuntan Sussman et al. (2011) se relaciona con la génesis de ciertas adiciones.

En línea con la literatura comentada, sería esperable encontrar mayor relación entre usos de la red más cercanos a la comunicación con los contactos y las tendencias adictivas que otros usos más de carácter individualista -colgar comentarios personales o fotografías-, aunque hasta la fecha esta relación no ha sido contrastada.

\section{El papel de los padres en el uso de las redes sociales}

Una figura importante en la regulación de los usos de medios digitales es la de los padres (O'keeffe et al., 2011). Mientras los padres parecen mostrar cierto temor al uso de Internet, los adolescentes parecen mostrar una preocupación mucho menor (Livingstone, 2008). Diversos estudios han mostrado discrepancias en cuanto a la percepción de riesgo por parte de padres e hijos, y cómo estos últimos no intuyen peligro en algunas prácticas de riesgo tales como contactar con desconocidos, mostrar datos privados o hacer una utilización predominante de la red para el manejo de sus relaciones sociales (Lenhart y Madden, 2007; Liau, Khoo y Ang, 2008; McCarty et al., 2011). Los padres adquieren un doble rol en la relación de sus hijos con las redes sociales, pues por una parte pueden servir como reguladores de su uso (Liau et al., 2008), pero, además, también formar parte de su red de contactos (Kart y Peluchette, 2011; West, Lewis y Currie, 2009). 
Estudios previos han mostrado que los padres, al intentar ejercer una vigilancia o control sobre el uso que sus hijos realizan de Internet, tienden a infravalorar los riesgos y peligros que estos asumen. Como muestra el trabajo de Liau, Khoo y Ang (2008), los padres tienen una percepción errónea tanto del uso y tiempo de uso que sus hijos dan a la red, como de las prácticas de control parental que ponen en práctica. Los hijos manifestaron en este estudio, pasar casi el doble de horas conectados a Internet que lo que sus padres percibían, y acceder 4 veces más a contenidos violentos y a prácticas de riesgo como quedar con desconocidos. Unido a esta distorsión en la percepción del uso, la comunicación entre padres e hijos sobre un uso inapropiado de Internet es escasa (apenas un 33\%). Este hecho es especialmente preocupante, al estar relacionada una mala comunicación entre padres e hijos con un mal uso de los entornos digitales (Mesch, 2003).

Existe cierto consenso entre los investigadores al afirmar que las madres ejercen un papel más activo en la supervisión del uso de Internet. Las madres no solo conocen mejor que los padres la vida de los adolescentes (Bumpus, Crouter y McHale, 2001; Waizenhofer, Buchanan y Jackson-Newsom, 2004), sino que pasan más tiempo compartiendo actividades y hablando con ellos (Crouter y McHale, 1993). Son más conscientes de los peligros a los que sus hijos se exponen en la red (Liau et al., 2008) y están más dispuestas a conseguir información sobre los usos que estos hacen en Internet.

Este posible papel protector de las madres, no obstante, no ha sido comprobado de manera empírica en ningún estudio previo, por lo que parece pertinente comprobar en qué medida la presencia de la madre en la red social puede ser un elemento "protector" de un uso adictivo a la misma.

\section{El presente estudio}

Este estudio examina el uso que jóvenes españoles hacen de la red social Tuenti, y su posible relación con las tendencias adictivas a la misma. En este sentido, en línea con la literatura comentada, esperamos que aquellos usos de la red Tuenti más cercanos a la comunicación directa con los contactos se relacionen en mayor medida con las tendencias adictivas informadas por los jóvenes (Hipótesis 1). En segundo lugar, se analiza el posible rol protector que la figura materna puede desempeñar respecto a las tendencias adictivas mostradas por los jóvenes, de tal forma que esperamos que la actitud positiva de estos hacia la presencia de su madre en la red social Tuenti repercuta en una menor tendencia adictiva informada (Hipótesis 2). En tercer lugar, teniendo en cuenta los hallazgos de diferentes estudios que sugieren la existencia de diferencias de género en los usos adictivos de las redes sociales, esperamos que los chicos muestren mayores tendencias adictivas a la red Tuenti que las chicas (Hipótesis 3). Finalmente, esperamos también encontrar diferencias en la relación entre usos y tendencias adictivas en función del género, de modo que la relación entre usos y adicción sea mayor en los chicos que en las chicas (Hipótesis 4).

\section{Método}

\section{Participantes}

Participaron en este estudio 221 estudiantes universitarios de diferentes titulaciones de la Universidad de Sevilla, de los que veinticinco fueron eliminados de este estudio al no poseer cuenta en la red social Tuenti. Finalmente, la muestra quedó compuesta por 143 mujeres y 56 hombres. Su rango de edad osciló entre los 18 y los 22 años, siendo la media para las mujeres de 19.09 años de edad $(D T=1.29)$ y para los hombres de 19.19 años de edad $(D T=1.28)$.

\section{Materiales}

Se elaboró un cuadernillo que recogía información acerca del nivel académico y edad de los y las participantes, así como diferentes ítems dirigidos a recabar información sobre:

Usos de la red social Tuenti. Un total de 33 ítems recababan información acerca de los posibles usos que hacen los jóvenes de la red social Tuenti, por ejemplo: "utilizo la red social Tuenti para comunicarme a través del chat con mis amigos", "utilizo la red social Tuenti para compartir vídeos y música con mis amigos", "utilizo la red social Tuenti para discutir temas académicos", "utilizo la red social Tuenti para conocer las actividades de mis amigos". Estos ítems tenían una opción de respuesta de 6 puntos, siendo " 0 " (totalmente en desacuerdo) a "5" (totalmente de acuerdo).

Adicción a la red social Tuenti. Para evaluar el nivel de adicción a la red social Tuenti se adaptaron los 10 ítems del Cuestionario de Experiencias Relacionadas con Internet CERI- (Beranuy, Chamarro, Graner y Sánchez-Carbonell, 2009), previamente utilizado por Gracia, Vigo, Fernández y Marcó (2002) y posteriormente por Carbonell et al. (2012). Los 7 primeros ítems, por ejemplo, "cuando tengo problemas, conectarme a la red social Tuenti me ayuda a evadirme de ellos", "cuando me conecto a Tuenti, me pasa el tiempo sin darme cuenta", "cuando no estoy conectado a Tuenti, me siento agitado/a o preocupado/a", presentaban una escala de respuesta tipo Likert de 6 puntos $(0$, totalmente en desacuerdo;5, totalmente de acuerdo), mientras que la escala de respuesta de los tres últimos ítems era de 4 puntos (1, nunca; 4 , siempre). La consistencia interna para esta escala fue de $\alpha=.81$.

Actitudes hacia la presencia de los padres en la red social Tuenti. Se diseñaron dos preguntas dirigidas a recoger las actitudes de los participantes hacia la presencia de sus padres dentro de su red de contactos Tuenti: "agregaría a mi padre como contacto Tuenti", "agregaría a mi madre como contacto Tuenti”. Se adoptó una escala de respuesta tipo Likert de 6 puntos, desde " 0 " (totalmente en desacuerdo) hasta " 5 " (totalmente de acuerdo). 


\section{Procedimiento}

Los datos se recogieron durante el mes de Mayo de 2011. Los cuestionarios fueron administrados por los autores del estudio en las aulas de los universitarios al finalizar una de sus clases (aproximadamente durante 10 minutos). Se preguntó al alumnado si era usuario de la red social Tuenti. A quienes contestaban de forma afirmativa se les entregaba el cuadernillo. Las instrucciones verbales y escritas del estudio explicitaban el carácter voluntario de la participación, y garantizaban el anonimato y confidencialidad de las respuestas.

\section{Resultados}

\section{Análisis factorial}

El primer objetivo que nos planteábamos con este estudio era examinar el uso que jóvenes españoles hacen de la red social Tuenti. Para identificar la estructura subyacente a los usos de la red social Tuenti, se realizó un análisis factorial ex- ploratorio con rotación Varimax. Con el objetivo de verificar la idoneidad de la matriz de datos para el análisis factorial, antes de realizar el mismo calculamos el coeficiente KaiserMeyer-Olkin (KMO) (Sharma, 1996). Los resultados de esta prueba fueron satisfactorios $\left(K M O=.75, \chi^{2}\right.$ Barlett $(496)=$ 3141.4; $p<.001)$. El análisis factorial mostró la existencia de 4 factores que explicaban el $47.02 \%$ de la varianza. El primer factor (Entretenimiento) incluyó 8 ítems que explicaban el $13.13 \%$ de la varianza, el segundo factor (Comunicación a través del muro) incluyó 6 ítems que explicaban el 12.08\% de la varianza, el tercer factor (Comunicación privada) incluyó 13 ítems que explicaban el $11.13 \%$ de la varianza, y el último factor (Información sobre actividades sociales) incluyó 5 ítems que daban cuenta del $10.68 \%$ de la varianza (Tabla 1 ). La consistencia interna mostrada para la escala completa fue de $\alpha=.89$. El primer factor mostró una consistencia interna de $\alpha=.83$, el segundo de $\alpha=.87$, el tercero de $\alpha=.74$ y el último factor de $\alpha=.74$.

Tabla 1. Análisis factorial de los usos de la red social Tuenti.

\begin{tabular}{|c|c|c|c|c|}
\hline & \multicolumn{4}{|c|}{ Factores } \\
\hline & 1 & 2 & 3 & 4 \\
\hline \multicolumn{5}{|l|}{ Items: Utilizo Tuenti para... } \\
\hline \multicolumn{5}{|l|}{ Factor 1. Entretenimiento } \\
\hline Ver videos subidos por mis amigos & .78 & .19 & .06 & .16 \\
\hline Escuchar música subida por mis amigos & .77 & .21 & .04 & .17 \\
\hline Compartir videos y música con mis amigos & .74 & .28 & .01 & .13 \\
\hline Ver videos subidos por mis contactos & .71 & .28 & .08 & .33 \\
\hline Compartir videos y música con mis contactos & .60 & .29 & .09 & .29 \\
\hline Escuchar música subida por mis contactos & .58 & .01 & .05 & .10 \\
\hline Subir fotos de mis amigos & .51 & .16 & .37 & .03 \\
\hline Subir fotos personales & .50 & .05 & .41 & -.05 \\
\hline \multicolumn{5}{|l|}{ Factor 2. Comunicación a través del muro } \\
\hline Escribir en el muro de mis amigos & .20 & .78 & .10 & -.04 \\
\hline Escribir en el muro de mis contactos & .22 & .76 & .24 & .09 \\
\hline Leer el muro de mis amigos & .08 & .73 & .14 & .26 \\
\hline Leer mi muro & .17 & .71 & .13 & .01 \\
\hline Leer el muro de mis contactos & .09 & .70 & .14 & .38 \\
\hline Escribir en mi muro & .29 & .65 & .09 & -.08 \\
\hline \multicolumn{5}{|l|}{ Factor 3. Contacto privado } \\
\hline Enviar mensajes privados & .08 & -.02 & .65 & .04 \\
\hline Pedir/dar consejo sobre la realización de actividades académicas & -.23 & .13 & .59 & .08 \\
\hline Conocer los hábitos/actividades de mis amigos & .20 & .30 & .55 & .17 \\
\hline Comunicarme a través del chat con mis contactos & .20 & .01 & .50 & -.01 \\
\hline Comunicarme a través del chat con mis amigos & .24 & .06 & .50 & -.21 \\
\hline Discutir temas académicos & .21 & .17 & .50 & .14 \\
\hline Enviar mensajes privados en vez de mi cuenta de email & -.04 & .09 & .48 & -.08 \\
\hline Conocer los hábitos/actividades de mis contactos & .06 & .26 & .47 & .37 \\
\hline Comentar con mis amigos cosas que me pasan & -.01 & .29 & .44 & .09 \\
\hline Felicitar a mis amigos por sus cumpleaños & .20 & -.06 & .43 & .31 \\
\hline Felicitar a mis contactos por sus cumpleaños & .26 & -.06 & .42 & .32 \\
\hline Comentar las fotos, estados, opiniones de mis contactos & .26 & .17 & .36 & .30 \\
\hline Comentar con mis amigos noticias que he visto en periódicos, telediarios, etc & .07 & .13 & .30 & .18 \\
\hline \multicolumn{5}{|l|}{ Factor 4. Información sobre actividades sociales } \\
\hline Estar informado sobre actividades sociales programadas por mis contactos & .05 & .05 & -.02 & .81 \\
\hline Estar informado sobre actividades sociales programadas por mis amigos & .09 & .19 & -.04 & .72 \\
\hline Invitar a mis contactos a actividades sociales & .29 & -.01 & .16 & .71 \\
\hline Invitar a mis amigos a actividades sociales & .28 & .05 & .12 & .64 \\
\hline Unirme a actividades sociales programadas por amigos y contactos & .06 & .04 & .01 & .50 \\
\hline Valor & 4.20 & 3.87 & 3.56 & 3.42 \\
\hline Varianza explicada (\%) & 13.13 & 12.08 & 11.13 & 10.68 \\
\hline
\end{tabular}




\section{Correlaciones}

En relación con la primera hipótesis de nuestro estudio, se exploró si los diferentes usos que hacen los jóvenes universitarios de la red social Tuenti podrían estar relacionados con las tendencias en los niveles de adicción informados por los mismos a dicha red. Además, también exploramos si las actitudes hacia la presencia de sus padres entre sus contactos Tuenti podrían mostrar alguna relación con sus tendencias adictivas a la red social. Para ello, en primer lugar se llevó a cabo un análisis de correlaciones entre todas estas variables del estudio, y posteriormente un análisis de regresión. La Tabla 2 recoge las correlaciones halladas entre los cuatro usos identificados por el análisis factorial de la red social Tuenti, la escala de tendencias adictivas a Tuenti y las actitudes de los jóvenes hacia la presencia de sus progenitores en su red de contactos Tuenti.

Tabla 2. Estadísticos descriptivos y correlaciones entre las principales variables del estudio.

\begin{tabular}{|c|c|c|c|c|c|c|c|c|c|}
\hline$\overline{\text { Variable }}$ & $M$ & $D T$ & 1 & 2 & 3 & 4 & 5 & 6 & 7 \\
\hline 1. Entretenimiento & 2.77 & 1.14 & 1 & $.46^{* *}$ & $.37 * *$ & $.39 * *$ & .04 & .04 & $.25 * *$ \\
\hline 2. Comunicación Muro & 2.64 & 1.11 & & 1 & $.45^{* *}$ & $.26 * *$ & -.05 & -.05 & $.32 * *$ \\
\hline 3. Comunicación privada & 3.30 & .80 & & & 1 & $.30 * *$ & -.05 & -.03 & $.34 * *$ \\
\hline 4. Actividades Sociales & 3.13 & 1.20 & & & & 1 & -.09 & -.06 & $.22 * *$ \\
\hline 5. Aceptación Madre & 1.86 & 2.02 & & & & & 1 & $.92 * *$ & $-.16^{*}$ \\
\hline 6. Aceptación Padre & 1.74 & 2.00 & & & & & & 1 & -.13 \\
\hline 7. Adicción & 1.44 & .79 & & & & & & & 1 \\
\hline
\end{tabular}

$\frac{7 . \text { Adiccion }}{* p<.05 ;{ }^{* *} p<.01}$

Como podemos apreciar en la Tabla 2, se encontró una correlación moderada y de tipo positivo entre cada uno de los usos identificados y los niveles de adicción a Tuenti. En concreto, la correlación más alta fue la mostrada por Comunicación privada $(r=.34, p<.01)$, seguida de la mostrada por la Comunicación a través del muro $(r=.32, p<.01)$, Entretenimiento $(r$ $=.25, p<.01)$ e Información sobre actividades sociales $(r=.22, p<$ .01). Además, este análisis evidenció la existencia de una relación de tipo negativo entre las actitudes hacia la aceptación de la madre (pero no del padre) en la red Tuenti y los niveles de adicción $(r=-.16, p<.05)$.

\section{Análisis de regresión}

A continuación, llevamos a cabo un análisis de regresión jerárquica para evaluar si las variables usos de la red social Tuenti, actitudes hacia la aceptación de los padres, y género de los participantes mostraban relaciones independientes con la principal variable dependiente de este estudio (tendencias adictivas a la red social Tuenti). Los principales resultados se pueden ver en la Tabla 3.

Tabla 3. Regresión sobre la variable tendencias adictivas a la red social Tuenti.

\begin{tabular}{|c|c|c|c|c|c|}
\hline Variable & $\Delta \mathrm{R}^{2}$ Ajust. & $\Delta F$ & Error tip. & Beta & $t$ \\
\hline Paso 1 & .20 & $7.50^{* *}$ & .66 & & \\
\hline Comunicación Privada & & & .07 & .20 & $2.57^{*}$ \\
\hline Aceptación madre & & & .02 & -.16 & $-2.35^{*}$ \\
\hline Paso 2 & .06 & .98 & .72 & & \\
\hline Comunicación Privada x Género & & & .18 & .67 & $2.16^{*}$ \\
\hline
\end{tabular}

En el primer paso se introdujeron todas las variables (Entretenimiento, Comunicación a través del muro, Comunicación privada, Información sobre actividades sociales, Actitudes hacia la aceptación del padre y de la madre, y Género), y en el segundo paso, se introdujeron las interacciones de segundo orden. En el primer paso, este análisis mostró un efecto principal de la variable Comunicación privada $(\beta=$ $.20, t=2.57, p<.05)$ y de la actitud positiva hacia la aceptación de la madre como contacto Tuenti $(\beta=-.16, t=-2.35$, $p$ $<.05)$, así como una tendencia marginalmente significativa de la variable comunicación a través del muro $(\beta=.15, t=$ $1.85, p=.06)$, sobre los niveles de adicción informados por los participantes. Ninguna otra variable alcanzó la significación estadística. En el caso de la primera variable, esta relación indica que los mayores niveles de adicción a la red social Tuenti son mostrados por aquellos y aquellas participantes que informan de un contacto más privado con sus amigos a través de la red Tuenti (por ejemplo, utilizando el chat de la red Tuenti para hablar con los contactos o haciendo uso del envío de mensajes privados a través de esta red en lugar de hacerlo por el sistema tradicional de correo electrónico). Por el contrario, aquellos estudiantes que mostraban una actitud más positiva hacia la presencia de su madre entre sus contactos de la red Tuenti (es decir, reconocían abiertamente que aceptarían a su madre como contacto Tuenti) informaban de menores niveles de adicción a Tuenti que aquellos participantes cuyas actitudes hacia la aceptación de la madre no eran favorables.

Sin embargo, en el segundo paso de la regresión, obtuvimos una interacción significativa entre las variables Comunicación privada y Género $(\beta=.68, t=1.93, p=.05)$, por lo que la interpretación de la primera variable sobre los niveles 
de adicción a la red social Tuenti necesita ser interpretada teniendo en cuenta el efecto del Género de los participantes (Figura 1).

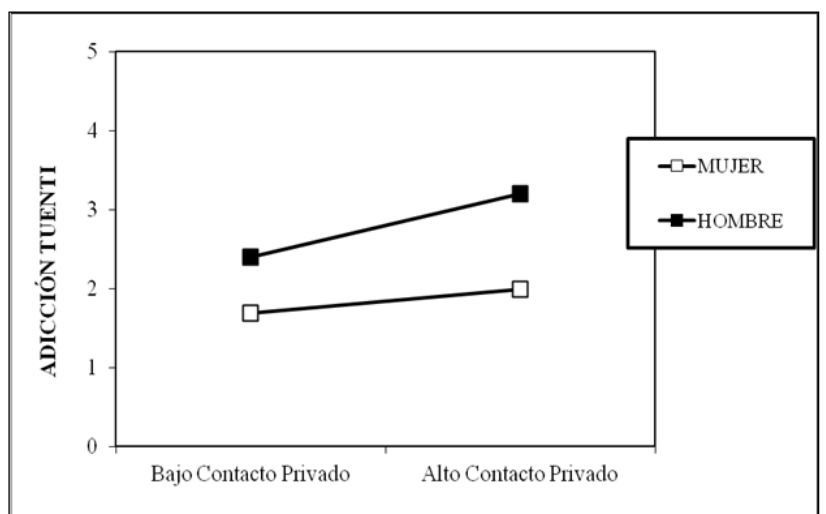

Figura 1. Relación entre el uso "Contacto privado" y adicción a la red social Tuenti en función del Género de los participantes.

Analizando esta interacción, los análisis post-hoc mostraron, como podemos observar en la Figura 1, que en el caso de las mujeres sus tendencias adictivas a la red social Tuenti no difieren en función del uso que realizan de la red Tuenti para mantener un alto o bajo contacto íntimo con sus amigos, $t(6,50)=1.81 ; p=n s$, sin embargo, en el caso de los hombres, los niveles de adicción informados son significativamente superiores cuando mantienen un alto contacto privado con sus amigos, $t(53)=5.01 ; p<.001$, que cuando este contacto es bajo.

\section{Discusión}

El objetivo de este estudio ha sido analizar, desde el paradigma de la teoría de usos y gratificaciones, el uso que los jóvenes universitarios españoles dan a la red social Tuenti, y en qué medida el tipo de uso se asocia con las tendencias adictivas a la red Tuenti. Además se analizó el papel que los progenitores tienen como facilitadores o inhibidores de dichas tendencias y el impacto del género de los participantes.

Los resultados de este estudio muestran la existencia de 4 categorías diferenciadas en el uso que estos jóvenes dan a la red social Tuenti. Así se utiliza esta red social como fuente de entretenimiento (Factor 1), como herramienta de comunicación con sus amigos, ya sea a nivel público través del muro (Factor 2) o a un nivel más privado mediante otras herramientas (Factor 3) como el uso del chat o la creación de comentarios, y como fuente de información social (Factor 4), tanto para informar sobre actividades sociales en las que se participa como para poder seguir la actividad social de los contactos o amigos. Los usos de esta red son, en parte, coincidentes con los encontrados en otras redes sociales, en las que también se detecta un uso vinculado al entretenimiento (e.g. Cheung et al., 2010), y a la posibilidad de estar en contacto con otras personas (Raacke et al., 2008; Subrahmanyam et al., 2008). El hecho de poder compartir información y re- cursos con otros contactos a través de la red social, es también un mecanismo que facilita la socialización (Park et al., 2009), y la intención de seguir utilizando la red social (Cheung et al., 2010).

De entre los usos que los jóvenes dan a la red social Tuenti, la comunicación privada -aquella que utiliza vías de comunicación como el chat o mensajes personales- parece estar relacionada con un incremento de la tendencia adictiva en el uso de la red social, mostrando apoyo a nuestra Hipótesis 1. En este sentido, algunos estudios (e.g., Carbonell et al., 2012) ya habían sugerido que la utilización de vías de comunicación que permiten una alteración de la identidad se relaciona con el riesgo de padecer una adición. Aunque las redes sociales muestran un único perfil de usuario, es posible que el mismo perfil muestre diferentes niveles de concreción. Así, como apunta Livingstone (2008), algunos usuarios pueden dotar de muchos detalles a su perfil, utilizando el mismo como herramienta de autoafirmación, pero otros pueden mostrar información opaca o en ocasiones cambiante. La investigación futura debería indagar en este aspecto y explorar si los posibles niveles de concreción de los perfiles de usuario pueden suponer una alteración de la identidad de la persona y si esto podría estar relacionado con su nivel de adicción a las redes sociales.

Además, como indican los resultados, esta relación entre un uso privado de la red con los contactos y la tendencia adictiva, es especialmente relevante en el caso de los chicos, en comparación con las chicas, mostrando apoyo a la Hipótesis 4. Los chicos muestran una mayor tendencia adictiva a la red social Tuenti cuando realizan un mayor uso de la red con fines de comunicación privada con sus contactos, frente a aquellos chicos que realizan un uso privado de la misma menos frecuente. En el caso de las chicas apenas existen diferencias entre usar o no este tipo de comunicación privada con los contactos a través de Tuenti, siendo en todo caso sus niveles de tendencia a la adicción inferiores a los de los chicos. En este sentido, nuestros resultados coinciden con los de la literatura previa (e.g. Beranuy et al., 2009) que ya han mostrado que son los chicos, en comparación con las chicas, los que con mayor frecuencia exhiben patrones de adicción a Internet (Carbonell et al., 2012, para una revisión). La investigación futura deberá determinar si estas diferencias de género en niveles de adicción a Internet se encuentran también de manera sistemática en otras redes sociales, y en diferentes grupos de edad. En su conjunto, estos resultados apoyan la Hipótesis 3 de este estudio, al mostrar que existen diferencias en el uso que chicas y chicos realizan de las redes sociales, y al resaltar que los chicos pueden tener una mayor tendencia que las chicas a realizar un uso no adecuado de las redes sociales.

En relación al papel de los padres, la actitud positiva manifestada por los y las jóvenes hacia la presencia de la figura de la madre en la red social Tuenti se dibuja como un factor de protección de las tendencias adictivas de los jóvenes a la red social, encontrando con ello apoyo a la Hipótesis 2 de este estudio. Estos resultados van en la misma línea de aquellos 
que también encuentran diferencias entre padres y madres relacionadas con las nuevas tecnologías (Bjornstad y Elligsen, 2004; Bumpus et al., 2001; Waizenhofer et al., 2004). Así, la posibilidad de contar con la madre como contacto se relaciona con una reducción de la tendencia adictiva informada por los jóvenes, pero no se encuentra efecto alguno cuando el contacto es el padre. Este hallazgo ofrece nuevas oportunidades en el contexto del control parental. Tal vez no se trata tanto de limitar el acceso a Internet sino de poder estar presente, desde el mismo medio, ofreciendo nuevas vías de comunicación. Como ya han indicado Liau y colaboradores (2008) las estrategias de control basadas en la mejora de la comunicación han mostrado ser más eficaces que las basadas en elementos restrictivos.

Este trabajo adolece de algunas limitaciones. Para empezar, se ha utilizado de manera exclusiva la metodología de autoinforme para la evaluación de los usos de la red social Tuenti por parte de los jóvenes universitarios. En este sentido, habría sido útil la realización de grupos de discusión que complementaran este tipo de información aportada por el alumnado universitario. Futuras investigaciones deberían incluir en sus estudios, ambas metodologías de recogida de información. Además, los resultados de este trabajo se derivan de población universitaria, en la que ha sido mayoritaria la participación de chicas, por lo que deberíamos ser cautos en la generalización de los resultados. Sería interesante realizar este tipo de estudios con otras muestras de población no universitaria, y de diferentes edades, en las que se equipare de manera intencional la distribución de chicos y chicas. Por otro lado, es importante señalar que este estudio se centra en la intención manifestada por los universitarios de vincular a sus progenitores como contactos en su cuenta de Tuenti, pero no investiga si estos ya forman parte o no de dicha red. El interés de este estudio se centra en la actitud de los jóvenes hacia la presencia de sus padres como contacto en la red

\section{Referencias}

Ancu, M. y Cozma, R. (2009). MySpace Politics: Uses and Gratifications of Befriending Candidates. Journal of Broadcasting and Electronic Media, 53, 567-583.

Bargh, J. A. y McKenna, K. Y. A. (2004). The Internet and social life. Annual Review of Psychology, 55, 573-590.

Becoña, E. (2006). Adicción a nuevas tecnologías. Vigo: Nova Galicia Edicións

Beranuy, M., Chamarro, A., Graner, C. y Carbonell, X. (2009). Validación de dos escalas breves para evaluar la adicción a Internet y el abuso de móvil. Psicothema, 21, 480-485.

Bumpus, M. F., Crouter, A. C. y McHale, S. M. (2001). Parental autonomy granting during adolescence: Exploring gender differences in context. Development Psychology, 37, 163-173.

Carbonell, X., Chamarro, A., Griffiths, M., Oberst, U., Cladellas, R. y Talarn, A. (2012). Uso problematico de Internet y móvil en adolescentes y jóvenes españoles. Anales de Psicología, 28, 789-796.

Carbonell, X., Fúster, H., Chamorro, A. y Oberst, U. (2012). Adicción a Internet y móvil: Una revisión de estudios empíricos españoles, Papeles del Psicólogo, 33, 82-89.

Cheung, C. M. K., Chiu P. Y. y Lee, M. K. O. (2010). Online social networks: Why do students use facebook? Computers In Human Behavior, 27, 1337-1343.
Tuenti más que en el hecho manifiesto de tenerlos vinculados, ya que podría ser que contaran con su presencia aunque ésta no fuese deseada. Estudios futuros podrían contrastar si existen diferencias en las tendencias adictivas informadas por los jóvenes universitarios en función de si sus padres forman o no parte de su red de contactos. Por último, destacar la dificultad que supone la comparación de los resultados de este estudio con los de otros trabajos previos, ya que la investigación relacionada con esta red social es muy escasa.

A pesar de estas limitaciones, este trabajo realiza contribuciones que suponen un avance en el conocimiento de la relación de los jóvenes con la red social Tuenti. De entre las aportaciones, destacar en primer lugar que es el primer trabajo que analiza las tendencias adictivas en la red social Tuenti, ya que si bien es cierto que estudios precedentes (ver revisión de Carbonell et al., 2012) han analizado la adicción en Internet, estos no se ha centrado en dicha red social, sino en el uso general de Internet o de los móviles. En segundo lugar, ofrece una tipología específica del uso de Tuenti y da información clave para el desarrollo de programas preventivos de usos inadecuados. Y en tercer lugar, muestra datos empíricos que refuerzan la idea de que los progenitores desempeñan un papel diferenciado en la protección de un uso inadecuado de las redes sociales.

Futuros estudios, con el fin de profundizar en alguno de los hallazgos de este trabajo, deberían analizar cuál es la reacción emocional de los jóvenes cuando reciben una petición de "amistad" en las redes sociales por parte de sus progenitores, examinando si su aceptación podría estar condicionada por esta reacción. Esta información permitiría el diseño de programas de vigilancia y apoyo a un uso responsable de las redes sociales. Así mismo, sería interesante conocer si otras figuras de autoridad como profesores u otros familiares, tienen también alguna relación con el tipo de uso que realizan los jóvenes de las redes sociales.

Cheung, C. M. K. y Lee, M. K. O. (2009). Understanding the sustainability of a virtual community: Model development and empirical test. Journal of Information Science, 35, 279-298.

Chorng-Shyong, O., Shu-Chen, C. y Chih-Chien, W. (2011). Comparative loneliness of users versus nonusers of online chatting. Cyberpsychology, Behavior, and Social Networking, 14, 35-49.

Crouter, A. C. y McHale, S. M. (1993). Temporal rhythmic in family life: Seasonal variation in the relation between parental work and family processes. Developmental Psychology, 29, 198-205.

Ellison, N. B., Steinfield, C. y Lampe, C. (2007). The benefits of Facebook "friends": Social capital and college students' use of online social network sites. Journal of Computer-Mediated Communication, 12, 1143-1168.

Gentzler, A. L., Oberhauser, A. M., Westerman, D. y Nadorff, D. (2011). College students' use of electronic communication with parents: links to loneliness, attachment, and relationship quality. Cyberpsychology, Behavior, and Social Networking, 14, 71-74.

Gracia, M., Vigo, M., Fernández, M. J. y Marcó, M. (2002). Problemas conductuales relacionados con el uso de Internet: Un estudio exploratorio. Anales de Psicología, 18, 273-292.

Kalpidou, M., Costin, D. y Morris, J. (2011). The relationship between facebook and the well-being of undergraduate college students. Cyberpsychology, Behavior, and Social Networking, 14, 183-189. 
Karl, K. A. y Peluchette, J. V. (2011). "Friending” professors, parents and bosses: A Facebook connection conundrum. Journal of Education for Business, 86, 214-222.

Katz, E. (1959). Mass communication research and the study of popular culture: An editorial on a possible future for this journal. Studies in Public Communication, 2, 1-6.

Kim, J. H., Kim, M. S. y Nam, Y. (2010). An analysis of self-construals, motivations, facebook use, and user satisfaction. International Journal of $\mathrm{Hu}$ man-computer Interaction, 26, 1077-1099.

Kohut, A., Wike, R., Horowitz, J. M., Simmons, K., Poushter, J. y Barker, C. (2011). Global Digital Communication: Texting, Social Networking Popular Worldwide. Recuperado de http://www.pewglobal.org/2011/12/20/global-digitalcommunicationtexting-social-networking-popular-worldwide/.

Kujath, C. (2011). Facebook and myspace: complement o substitute for face-to-face interaction? Cyberpsychology, Behavior, and Social Networking, 14, 75-78.

Kuss, D. J. y Giffiths, M. D. (2011). Online Social Networking and Addiction-A Review of the Psychological Literature. International Journal of Environmental Research and Public Health, 8, 3528-3552.

Kwan, G. C. E. y Skoric, M. M. (2013). Facebook bullying: An extension of battles in school. Computers in Human Behavior, 29(1), 16-25.

Kwon, O. y Wen, Y. (2010). An empirical study of the factors affecting social network service use. Computers in Human Behavior, 26, 254-263.

Livingstone, S. (2008). Taking risky opportunities in youthful content creation: teenagers'use of social networking sites for intimacy, privacy and self-expression. New Media and Society, 10, 393-411.

LaRose, R. y Eastin, M. S. (2004). A social cognitive theory of Internet uses and gratifications: Toward a new model of media attendance. Journal of Broadcasting \& Electronic Media, 48, 358-377.

Lenhart, A. y Madden, M. (2007). Social networking websites and teens: An overview. Pew Internet \& American Life Project, Washington. Recuperado de http://www.pewinternet.org/pdfs/PIP SNS Data Memo Jan 2007. pdf.

Liau, A. K., Khoo A. y Ang, P. H. (2008). Parental Awareness and Monitoring of Adolescent Internet Use. Current Psychology, 27, 217-233.

Lowe, J. B., Barnes, M., Teo, C. y Sutherns, S. (2012). Investigating the use of social media to help women from going back to smoking postpartum. Australian and New Zealand Journal of Public Health, 36, 30-32.

Lyndon, A., Bonds-Raacke, J. y Cratt, A. (2011). College Students' Facebook Stalking of Ex-Partners. Cyberpsychology, Behavior, and Social Networking, 14, 711-716.

McCarty, C., Prawitz, A. D., Derscheid, L. E. y Montgomery, B. (2011). Perceived safety and teen risk taking in online chat sites. Cyberpsychology, Behavior, and Social Networking, 14, 174-184.

Mesch, G. S. (2003). The family and the Internet: The Israeli case. Social Science Quarterly, 84, 1038-1050.
Muise, A., Christofides, E. y Desmarais, S. (2009). More information than you ever wanted: Does Facebook bring out the greeneyed monster of jealousy? Ciberpsychology and Behaviour, 12, 441-444.

O'Keeffe, G. S. y Clarke-Pearson, K. (2011). Clinical Report - The Impact of Social Media on Children, Adolescents, and Families. Pediatrics, 127, 800-804

Ortega, R., Elipe, P., Mora-Merchán, J. A., Genta, M. L., Brighi, A., Guarini, A., Smith, P. K., et al. (n.d.). The emotional impact of bullying and cyberbullying on victims: a European cross-national study. Aggressive behavior, 38(5), 342-56.

Park, N., Kee, K. F. y Valenzuela, S. (2009). Being immersed in Social Networking Environment: Facebook groups, uses and gratifications, and social outcomes. Cyberpsychology, Behavior, and Social Networking, 12, 729733.

Raacke, J. y Bonds-Raacke, J. (2008). MySpace and Facebook: Applying the uses and gratifications theory to exploring friend-networking sites. $C y$ berpsychology, Behavior, and Social Networking, 11, 169-174.

Roberts, D. F., Foehr, U. G. y Rideout, V. (2005). Generation M: Media in the lives of 8-18 Year-olds. Menlo Park, Ca: Kaiser Family Foundation.

Ruggiero, M. T. (2000). Uses and gratifications theory in the 21 st Century. Mass Communication and Society, 3, 3-37.

Sanchez-Franco, M. J., y Roldan, J. L. (2010). Expressive aesthetics to ease perceived community support: Exploring personal innovativeness and routinised behavior as moderators in Tuenti. Computer in Human Behavior, 26, 1445-1457.

Severin, W. J. y Tankard, J. W. (1997). Communication theories: Origins, methods, and uses in the mass media (4th ed.). New York: Longman.

Sharma, S. (1996). Applied Multivariate Techniques. John Wiley and Sons, Inc: New York.

Subrahmanyam, K., Reich, S. M., Waechter, N. y Espinoza, G. (2008). Online and offline social networks: Use of social networking sites by emerging adults. Journal of Applied Developmental Psychology 29, 420-433.

Sussman, S., Leventhal, A., Bluthenthal, R.., Freimuth, M., Forster, M. y Ames, S. L. (2011). A framework for specificity of the addictions. International Journal of Environmental Research and Public Health, 8, 3528-3552.

Tokunaga, R. S. (2011). Friend me or you'll strain us: Understanding negative events that occur over social networking sites. Cyberpsychology, Behavior, and Social Networking, 14, 425-432.

Waizenhofer, R. N., Buchanan, C. M y Jackson-Newsom, J. (2004). "Mothers" and "fathers" knowledge of adolescents' daily activities: its sources and its links with adolescent adjustment. Journal of Family Psychology, 18, 348-360.

West, A., Lewis, J. y Currie, P. (2009). Students' Facebook “friends": Public and private spheres. Journal of Youth Studies, 6, 615-627.

Wright, M. F. y Li, Y. (2011). The associations between young adults' faceto-face prosocial behaviors and their online prosocial behaviors. Computer in Human Behavior, 27, 1959-1962.

(Articulo recibido: 17-9-2012; revisado: 25-2-2013; aceptado: 4-3-2013) 